\title{
On-line Measurement of Internal Resistance of Lithium Ion Battery for EV and its Application Research
}

\author{
Hua Zhang ${ }^{1,2}$ Rengui Lu ${ }^{1}$, Chunbo Zhu ${ }^{1}$ and Yongping Zhao ${ }^{1}$ \\ ${ }^{1}$ School of Electrical Engineering and Automation, \\ Harbin Institute of Technology, China \\ ${ }^{2}$ College of Electronic Science, Northeast Petroleum University, China \\ zhxueju@163.com
}

\begin{abstract}
Internal resistance of the battery can really reflect its own characteristics, which including health status of the battery, inconsistency, state of charge, thermal runaway. On-line measurement of internal resistance can real-time monitor the running state of each battery and accurately, can report fault state of the battery and handle fault in time. This paper implements the practical engineering module of on-line measurement of internal resistance of lithium ion battery for EV (Electric Vehicle) using AC analysis. The detection precision and stability of the module are less than 0.3\%. Based on experiments, the paper studies the stability and temperature characteristic of internal resistance of lithium battery. It also studies the relationship between the internal resistance and SOC, charging current with experiments. This paper analyzes the relationship between the internal resistance and default of the battery, such as inconsistency of batteries, excessive charging current, changing of work state, overcharging.
\end{abstract}

Keywords: lithium ion battery; On-line measurement of internal resistance; AC analysis; the property of internal resistance; fault diagnosis of battery

\section{Introduction}

As the power source and the carrier of energy of electric vehicles, battery plays a very important role in electric vehicle. Only in terms of cost, the price of battery accounts for $1 / 2$ of the EV's total price [1]. The battery can be equivalent to a voltage source with internal resistance, and also can be equivalent to a two-port device. In addition to voltage and current, the internal resistance is the important electrical parameter of the battery which indicates the performance of the battery.

The internal resistance of the battery includes the ohmic resistance and polarization resistance. Ohmic resistance is composed of electrode materials, electrolyte, separator resistance and contact resistance of each part. Polarization resistance is the resistance caused by polarization in electrochemical reaction, and polarization includes the electrochemical polarization and concentration polarization. Because of the complexity of the internal of the battery, batteries have different equivalent models in different applications. Only in ADVISOR there are five different equivalent models. Fig. 1 shows the common Thevenin's Equivalent circuit of batteries [2-4], where $I_{L}$ stands for the load current (taking the discharge current direction as the positive orientation), $\mathrm{U}_{\mathrm{t}}$ for terminal voltage of the battery, $\mathrm{U}_{\mathrm{oc}}$ for open circuit voltage of the battery, $R_{O}$ for the ohmic internal resistance, $R_{P}$ for the polarized internal resistance, and $\mathrm{C}_{\mathrm{P}}$ for the polarized capacitance. 
Internal resistance really reflects the state of health $[5,6]$, state of charge, and also can accurately monitor thermal runaway of the battery $[7,8]$. The detection of internal resistance increases the accuracy of the diagnosis of the inconsistency of batteries [9].

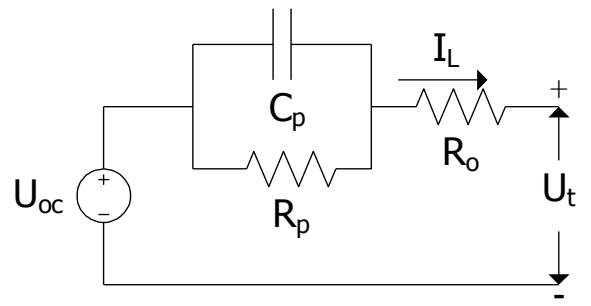

\section{Figure 1. The Common Thevenin's Equivalent Circuit of Batteries}

Although the internal resistance of the battery accurately reflects the work status and fault status of the battery, traditional internal resistance testing instrument cannot find the faulty battery in time because the measurement is regular and offline. On-line measurement system of internal resistance with high reliability can monitor the running state of each battery realtime and accurately. When the performance of single battery in the pack decreases greatly, measurement module informs the management system to take the corresponding operation in time. The measurement system can avoid rapid deterioration of the battery failure due to the time delay, even disastrous consequences.

The internal resistance of the battery really presents its characteristics, so the detection of variation of internal resistance increases the accuracy of battery failure diagnosis and also predicts the battery failure. Static detection of internal resistance can diagnose battery failure when the battery storing in exchanging battery station and when the electric vehicle in parking state. On-line measurement of internal resistance can diagnose battery failure when the electric vehicle is running.

Methods for the measurement of internal resistance of battery include DC discharge method and AC injection method [10]. Compared with the DC discharge method, the AC inserting method has the following advantages: (1) the measurement of the internal resistance of the battery is online, does not demand static or offline status. Therefore, the measurement avoids connection problems, and at the same time is able to get accurate and reliable measurement results. (2) The measurement avoids the accumulated damage to the battery performance caused by high-rate discharge compared with the DC discharge method, and does not affect the safe operation of energy store system. (3) The measurement does not require discharge load, and reduces the costs. Champlin proposed the method of DFIS (discrete frequency immittance spectroscopy) [11, 12], this method has better effect when applied to estimation of SOC and SOH $[13,14]$.This paper proposes a simple and reliable design as well as low costs.

\section{Module Design}

On-line measurement system of internal resistance is composed of three parts: On-line measurement module of internal resistance, USB-CAN, the software of the host. On-line measurement module of internal resistance can complete internal resistance measurement, internal resistance data coding, CAN data transmission and other functions. Because the computer have no CAN interface, the communication between On-line measurement module of internal resistance and host is realized based on USB-CAN. The software of the host can record, analyze and store the internal resistance data in real-time. 


\subsection{Principle of On-line Measurement Module of Internal Resistance}

This module selects the AC inserting method with the controlled current and puts forward improving the method, enhancing the ability of analog signal processing. When the controlled current $\Delta \mathrm{I}=\mathrm{I}_{\max } \sin (2 \pi f t)$ is injected into the two terminals of the battery, the voltage response is

Battery impedance is

$$
\Delta V=V_{\max } \sin (2 \pi f t+\phi)
$$

$$
Z(f)=\left(V_{\max } / I_{\max }\right) e^{j \phi}
$$

Equation (2) shows that the measurement results of battery impedance are dependent on frequency. The phase angle is $\emptyset$, and the impedance modulus is

$$
|Z|=V_{\max } / I_{\max }
$$

The real part of the impedance is

$$
|\mathrm{Z}| \cos \emptyset=\frac{\mathrm{V}_{\max }}{\mathrm{I}_{\max }} \cos \emptyset
$$

Assuming that the frequency of AC signal be injected into the battery is $\omega$, according to the simplified practical model of internal resistance of batteries as shown in fig. 1, the battery impedance is

$$
\mathrm{Z}=\mathrm{R}_{0}+\frac{\mathrm{R}_{\mathrm{p}}}{1+\omega^{2} \mathrm{C}_{\mathrm{p}}{ }^{2} \mathrm{R}_{\mathrm{p}}{ }^{2}}-\mathrm{j} \frac{\omega \mathrm{C}_{\mathrm{p}} \mathrm{R}_{\mathrm{p}}{ }^{2}}{1+\omega^{2} \mathrm{C}_{\mathrm{p}} \mathrm{R}_{\mathrm{p}}{ }^{2}}
$$

When the frequency of AC signal is injected into the battery stays between several hundred $\mathrm{Hz}$ and ten thousand $\mathrm{Hz}$, the curve of AC internal resistance of the battery is relatively stable [15]. So the frequency of measurement signal should be selected within this range.

Equation (4) shows that if $\omega$ is smaller, the imaginary part of the internal resistance is closer to zero, $\omega C_{p} R_{p}$ in real part is closer to zero. So it is more convenient to remove the effect of capacitance which makes the pure impedance approximating to $\left(R_{0}+R_{p}\right)$. In contrast, if $\omega$ is too big, the capacitance $\mathrm{C}$ has almost shortened, the measurement impedance values will completely ignore the polarized resistance $-R_{P}$, and it keeps the internal resistance of the battery closing to ohmic resistance $-\mathrm{R}_{\mathrm{O}}$. Experiment shows that the ohmic resistance of the cathode is an important part of the whole cell impedance; ohms resistance has influence on the battery capacity, the charge rate and discharge rate [16]. As shown above, the measurement module chooses $1 \mathrm{KHz}$ as the frequency of the AC signal injected into the battery.

According to national standard GB/T 15100-2003/IEC 61436:1998, the module of battery impedance is equivalent to the $\mathrm{AC}$ internal resistance of the battery. For this reason, this paper adopts internal resistance measurement through module of the impedance, which is achieved by calculating the peak of the voltage response of the battery.

\subsection{The Design of Measurement Module}

Figure 2 shows the circuit structure of an internal resistance measurement module. The sinusoidal voltage signal which is generated by the microprocessor unit is converted into a sinusoidal current signal with the same frequency and phase through the signal processing circuit and constant current source circuit, and outputs to the reference resistor and battery under test at the corresponding time. The sinusoidal voltage response across the reference resistor and the batteries is converted into a DC voltage signal through the amplifying circuit, the filter circuit and the peak holding circuit. DC voltage signal inputs to the microprocessor 
unit. The microprocessor unit compares the peak signal of the voltage response from the reference resistance with that of the battery measured, and calculates the internal resistance of the battery measured.

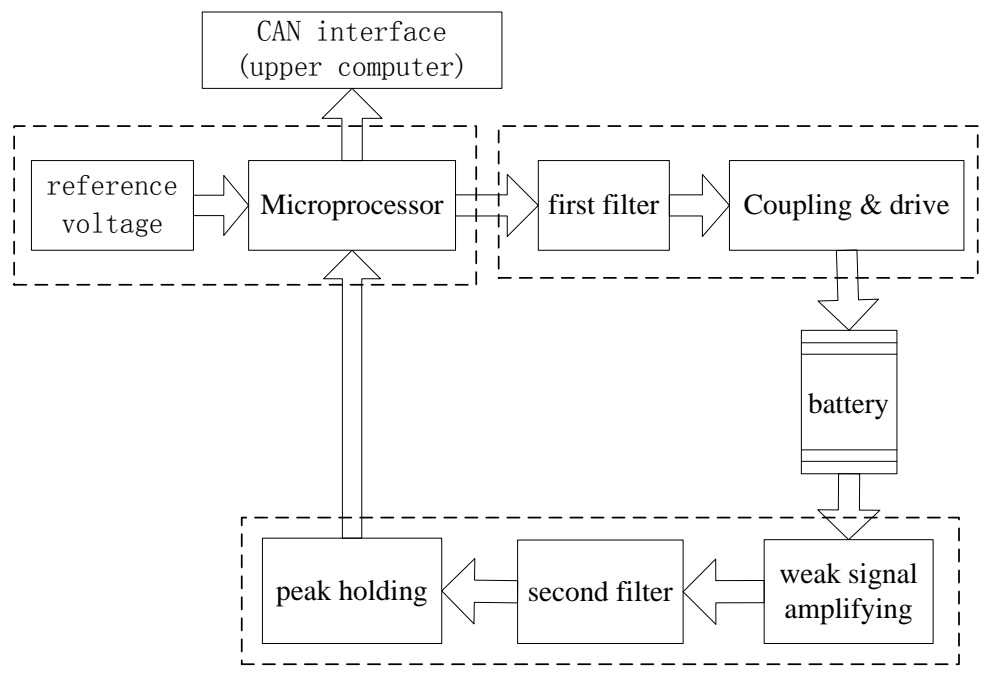

Figure 2. Circuit Structure of Internal Resistance Measurement Module

On-chip 12-bit D/A converter of the microprocessor unit generates $1 \mathrm{KHz}$ unipolar sinusoidal voltage signal. The signal is converted into a bipolar sinusoidal voltage signal through adder, and is input to the first filter followed. The microprocessor acquires the peak response of the battery through its internal 12 bit A/D converter, from which the internal resistance is obtained. The microprocessor also controls the constant current excitation signal and acquires peak signal to switch between the reference resistor and the battery under test.

The first and the second filter circuits are used to filter out the clutter and interference signal, and increase accuracy and stability of measurement. Filter circuit adopts a specific filter chip, and has the features of good dynamic characteristic, low noise, and high frequency precision.

Coupling and driving unit generates a stable, constant current sinusoidal drive current with $50 \mathrm{~mA}$, and the drive current is injected to reference resistance and battery under test. The constant current driving circuit adopts the improved HOWLAND constant current source circuit which is composed by high voltage, large current integrated operational amplifier and low noise integrated operational amplifier. The improved HOWLAND constant current source circuit has high output voltage flexibility, and inhibits the oscillation caused by resistor mismatch.

The internal resistance of the battery is at the order of $m \Omega$, as a result the voltage response signal of the internal resistance is at the order of $\mu \mathrm{V}$ and need be amplified with signal conditioning circuit for $\mathrm{AD}$ sampling. In the experiments the amplification effect of two cascaded amplifier circuits is better than that of high gain amplifier circuits composed by single operational amplifier, so amplifier circuit adopts two cascaded amplifier circuits respectively composed by precision instrumentation amplifier and low noise operational amplifier.

The peak holding circuit is composed of peak tracking circuit, peak holding capacitance and a peak discharge circuit. CAN interface unit encodes the internal resistance data acquired 
and transmits the result of internal resistance measurement. CAN interface is also responsible for communication with the host or the main control unit of battery management system.

Calibration of the result of internal resistance measurement is achieved by switching measurement circuit between reference resistance and the battery which is measured. In order to guarantee accuracy and stability of the sinusoid constant current source signal, the constant current source circuit employs high precision wire-wound resistor. The temperature drift of the wire-wound resistor is $25 \mathrm{ppm} /{ }^{\circ} \mathrm{C}$, with whose precision is $1 / 10000$. At the same time, the voltage response signal is measured using a four-wired for the purpose of reducing measurement error introduced by wires.

\subsection{The Software Design of Host}

The labview is used to program host software. In order to facilitate real-time recording, storage and analysis of the internal resistance data, software mainly consists of communication module, dynamic display module and data storage module.

Communication module receives the data which is sent by CAN bus of the internal resistance measurement module through the USB-CAN module, and decodes CAN bus data into internal resistance data according to the defined CAN protocol. The dynamic display module displays each measurement value of internal resistance and the overall variation curve in real time and dynamically in the host software interface. User can monitor the internal resistance and the variation tendency of internal resistance in real time. The data storage module stores the received impedance data and measurement time by excel form, facilitates the processing and analysis of resistance data.

\section{Experiment of Resistance Characteristics' Application in the Fault Diagnosis of the Battery}

An experimental bench has been built to verify the proposed system, as shown in Figure 3. The characteristics of internal resistance were analyzed through experiments, and the relationship between the internal resistance and failure of the battery was researched.

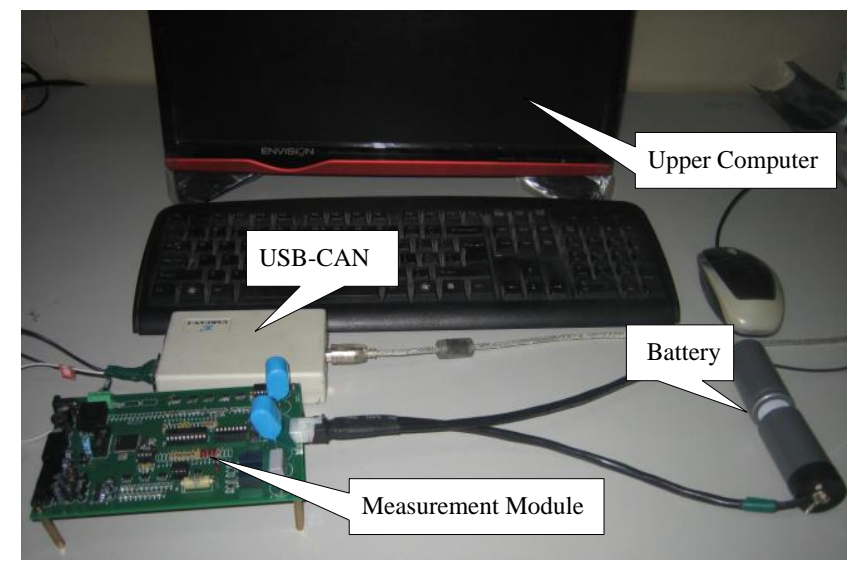

Figure 3 Experimental Platform for Prototype 


\subsection{The Relationship Between the Accuracy and Stability of Static Internal Resistance and Battery's Consistency}

Small resistances with the value of $12 \mathrm{~m} \Omega, 20 \mathrm{~m} \Omega$ and $30 \mathrm{~m} \Omega$ respectively, were measured by on-line measurement system of internal resistance and TH25121B intelligent DC low resistance tester. The accuracy of measurement of the resistance measurement module can be calculated by comparing the two measurement results. In the experiment, the same test point must be measured simultaneously with the two methods due to the effect of lead resistance and contact resistance. The measurement result of the intelligent DC low resistance tester was used as the reference value, and maximum relative error of measurement of the resistance is $0.29 \%$. Table 1 shows the comparison result of measuring accuracy from small resistance.

Table 1. The Comparison Result of Measuring Accuracy from Small Resistance

\begin{tabular}{lllll}
\hline unit $(\mathrm{m} \Omega)$ & $\begin{array}{l}\text { measurement } \\
\text { module }\end{array}$ & $\begin{array}{l}\text { measuring } \\
\text { instrument }\end{array}$ & $\operatorname{error}(\mathrm{m} \Omega)$ & $\begin{array}{l}\operatorname{accuracy}(\% \\
)\end{array}$ \\
\hline No. 1 resistance & 12.05 & 12.07 & -0.02 & -0.16 \\
\hline No. 2 resistance & 20.62 & 20.56 & 0.06 & 0.29 \\
\hline No. 3 resistance & 29.47 & 29.45 & 0.02 & 0.06 \\
\hline
\end{tabular}

Stability of measurement module can be obtained by measuring the internal resistance of the same battery continuously. The measurement system measured statically a certain brand of two 5Ah lithium iron phosphate battery 36 times continuously. The average of measurement is $22.87 \mathrm{~m} \Omega$, maximum absolute error is $0.06 \mathrm{~m} \Omega$, and maximum relative error of stability is $0.26 \%$.

Because the precision and stability of internal resistance measurement meet the requirements, the internal resistance's inconsistency of single battery can be detected. We can judge the battery's inconsistency based on a comprehensive consideration of inconsistency of internal resistance, voltage and SOC of a single battery. The faulty batteries with larger inconsistency could be picked out to avoid affecting the capacity and other performance of batteries. The battery can be judged been a short circuit fault when the resistance suddenly becomes very small. The battery connecting wire may be loose when the value of internal resistance vibrated in a range.

\subsection{The Relationship Between the Static Internal Resistance of Charged Battery and Temperature}

The following experiments were performed on two $5 \mathrm{Ah}$ lithium iron phosphate battery of a certain brand. The relationship between the static internal resistance and temperature in charging process is shown in Figure 4. At the same SOC, the static internal resistance of the battery decreases with the increase of temperature. In $-10^{\circ} \mathrm{C}$ to $20^{\circ} \mathrm{C}$, the rate of decline of internal resistance is fast, and the rate is $1.3 \mathrm{~m} \Omega / 10^{\circ} \mathrm{C}$. In $20^{\circ} \mathrm{C}$ to $50^{\circ} \mathrm{C}$, the rate becomes slow and is $0.42 \mathrm{~m} \Omega / 10^{\circ} \mathrm{C}$. The rate is about $1 / 3$ in the previous temperature interval. 


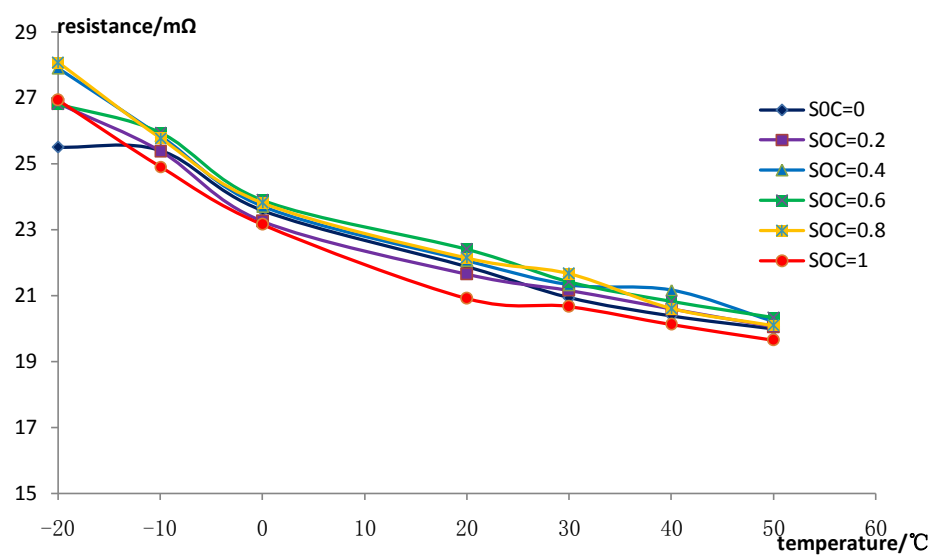

\section{Figure 4. The Relationship Between Static Internal Resistance of Charging State of the Battery and Temperature}

The change of internal resistance reflects the essential change of battery. The temperature change of batteries doesn't have special laws. But if the relationship between internal resistance and temperature changes sharply, or changes without the fixed rules, the battery must have faults. For example, the battery temperature changes abnormally, the temperature detection circuit of the BMS (battery management system) works abnormally. If the battery has a temperature fault, corresponding measures should be taken to batteries to avoid too high temperature or catching fire.

\subsection{The Relationship Between Static Internal Resistance of Charged Battery and} SOC

Figure 5 shows the relationship between static internal resistance of charged battery and SOC. We could draw a conclusion that under the same temperature static internal resistance of charged battery changes slightly with the increase of SOC, so static internal resistance of charged battery could not be as the judgment basis of SOC's reducing.

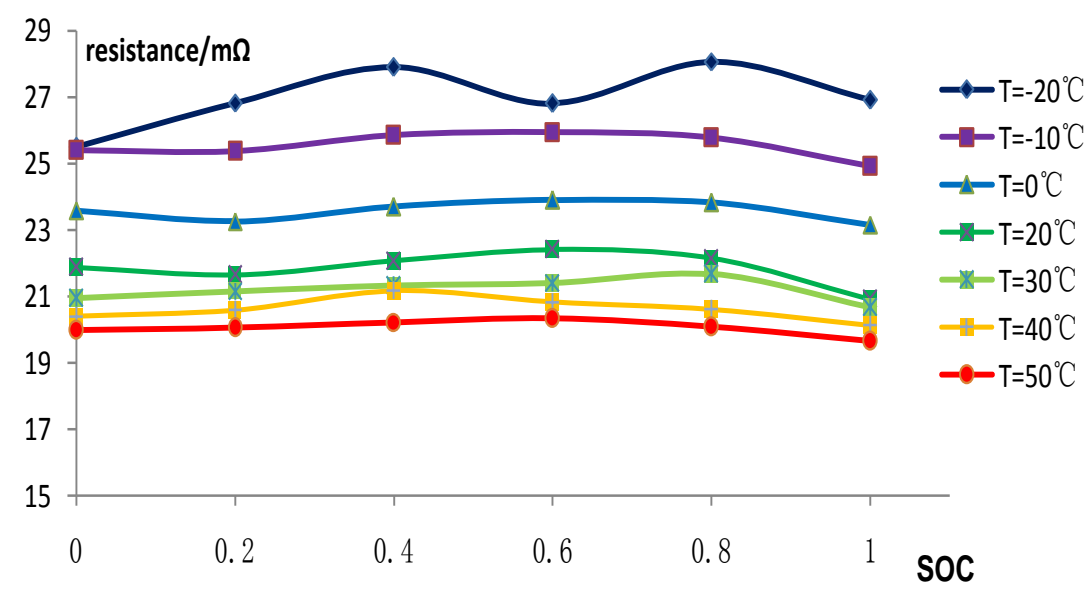

Figure 5. The Relationship Between Static Internal Resistance of Charged Battery and SOC 


\subsection{The Relationship Between On-line Internal Resistance of Charge Battery and Charging Current at Different Rates}

The relationship between on-line internal resistance of charged battery and charging current at different rates is shown in Figure 6. The internal resistance decreases with the increase of battery's SOC. The charging current is larger, internal resistance decreases more till the battery is fully charged, the descent speed of internal resistance is more rapid in the charge process, and under the same SOC the internal resistance is larger.

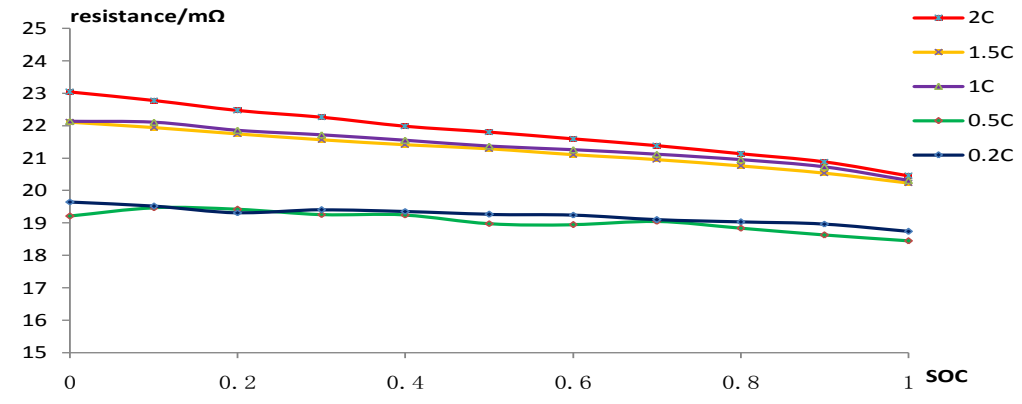

\section{Figure 6 The Relationship Between on-line Internal Resistance of Charged Battery and Charging Current at Different Rates}

Too large current would do irreversible damage to batteries, and even would cause too high temperature, catching fire and explosion. The relationship between internal resistance and SOC are different with different charging current. If the relationship between internal resistance and SOC changes sharply, or changes without the fixed rules, the fault of the battery must be determined. For example, the fault of current detection circuit of BMS, the fault of too large charging current.

\subsection{The Internal Resistance Change Curve in Charging-Discharging Process of Batteries}

Figure 7 shows the internal resistance change curve of a certain brand of two 5Ah lithium iron phosphate battery in charging-discharging process. Charge preparation process is static state, and the measurement result of internal resistance is stable. The maximum rate of change of resistance is $0.61 \%$ in this state.

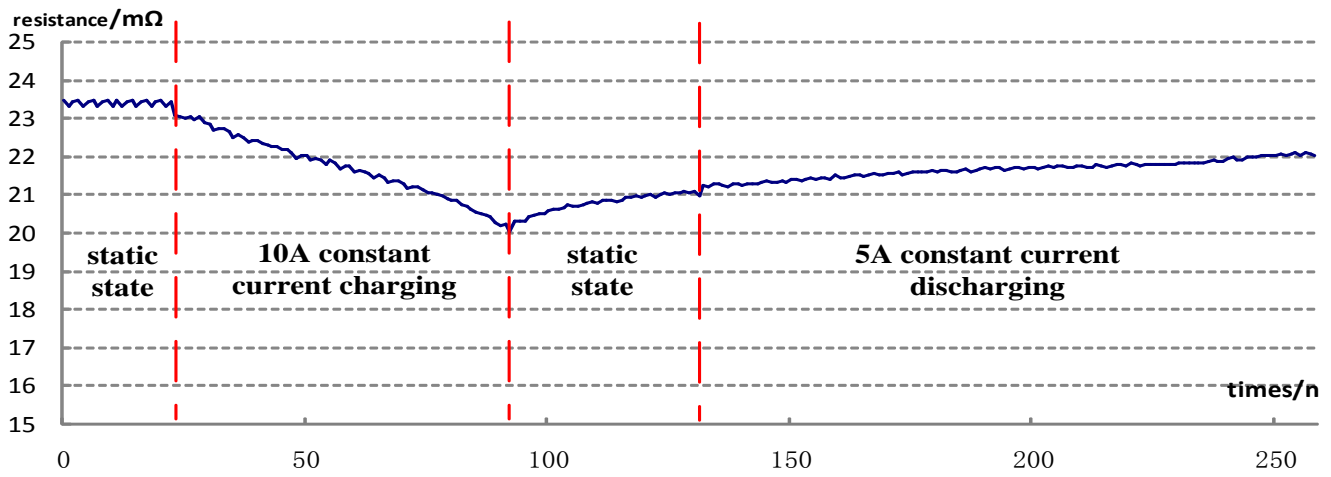

Figure 7. Internal Resistance Change Curve in Charging-discharging Process of Batteries 
In charge state, a great deal of lithium-ions strip from the cathode and enter into electrolyte because of the increasing of electrochemical activity of the battery. The resistance towards current of the battery is reduced and the internal resistance presents the decreasing tendency. The internal resistance reduces $12.3 \%$ when charging process completed comparing with the value before charging.

The battery stays in a static state after charging, the internal resistance of the battery rises gradually and the rising speed becomes more and more slowly in this state. The internal resistance keeps unchanged after the value reaches $21.1 \mathrm{~m} \Omega$. Subsequently, the battery is in discharging process with $1 \mathrm{C}$ constant current, and the internal resistance of the battery increases slowly.

The internal resistance of batteries would change sharply from static state to constant current charging state, from constant current charging state to static state, and from static state to constant current discharging state. So in the battery fault diagnosis system, the mutation of internal resistance could use to monitor the change of battery's working status.

\subsection{The Internal Resistance Change Curve in Overcharging Process of Batteries}

At room temperature, the battery charged with $1 \mathrm{C}$ constant current without setting the upper limit voltage. At the end of charging process the battery voltage was up to $9.21 \mathrm{~V}$, exceeded the upper cut-off voltage of $7.3 \mathrm{~V}$, the curve is shown in figure 8 .

At the initial static state the internal resistance of the battery is stable, and decreases at the $1 \mathrm{C}$ constant current charging stage. When the battery reaches the upper cut-off voltage and gets into the overcharge state, the decrease speed of internal resistance gets too large suddenly. According to this characteristic of lithium ion battery, the overcharge fault of the battery would be detected and forecasted.

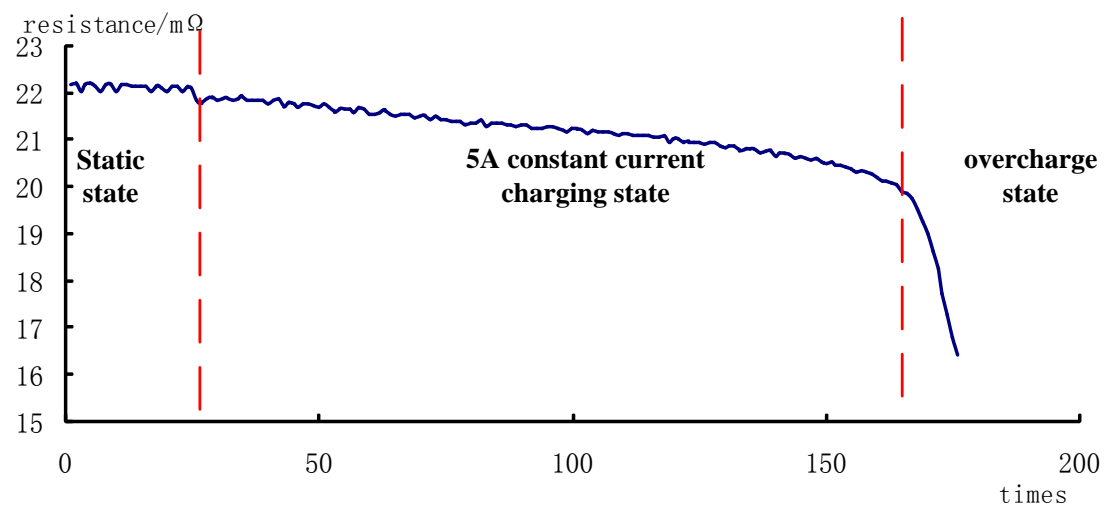

\section{Figure 8. The Internal Resistance Change Curve in Overcharging Process of Batteries}

\section{Conclusion}

This paper implements the practical engineering measurement system of internal resistance for lithium-ion battery which performs on-line measurement. Accuracy and stability of the measurement system are $0.29 \%$ and $0.26 \%$ respectively. This paper also studies the internal resistance's characteristics of lithium battery using the measurement system with experiments. The relationship between the internal resistance and the default of the battery has been analyzed, including inconsistency of batteries, excessive charging current, changing 
of work state, overcharging. On-line measurement system of internal resistance provides a new means for fault diagnosis of batteries besides monitoring of voltage, current and temperature of the battery manage system. It can also give new ideas for extremely dangerous fault diagnosis and performance prediction of batteries.

\section{References}

[1] X. Liu, "Battery Management System Design and Parameters Estimation Method Research", Master thesis, University of Science and Technology of China, (2011).

[2] H. Hongwen, X. Rui and G. Hongqiang, "Online estimation of model parameters and state-of-charge of LiFePO4 batteries in electric vehicles", Applied Energy, no. 89, no. 1, (2011), pp. 413-420.

[3] M. Verbrugge, "Adaptive", multi-parameter battery state estimator with optimized time-weighting factors, Journal of Applied Electrochemistry, vol. 37, no. 5, (2007), pp. 605-616.

[4] V. H. Johnson, "Battery performance models in ADVISOR", Journal of Power Sources, vol. 110, (2002), pp. 321-329.

[5] S. Pritpal and R. David, "Fuzzy Logic-based State-of-Health Determination of Lead Acid Batteries", 24th International Telecommunications Energy Conference, (2002), pp. 583-590, Montreal, Que, Canada.

[6] P. Singh, R. Vinjamuri, X. Wang and D. Reisner, "Design and Implementation of a Fuzzy Logic-based Stateof-Charge Meter for Li-ion Batteries Ued in Portable Defibrillators", Journal of Power Sources, (2006), vol. 162 , pp. 829-836.

[7] S. Rodrigues, N. Munichandraiah and A. K. Shukla, "AC impedance and state-of-charge analysis of a sealed lithium-ion rechargeable battery", J Solid State Electrochem, vol. 3, (1999), pp. 397-405.

[8] I. Uchida, H. Ishikawa, M. Mohamedi and M. Umeda, "AC-impedance Measurements During Thermal Runaway Process in Several Lithium/Polymer Batteries”, J Power Sources, vol. 119, (2003), pp.821-825.

[9] G. Ming, "Research on Battery Management System with Diagnostic Functions", Master thesis, Tsinghua University, (2009).

[10] K. R. Cooper and M. Smith, "Electrical test methods for on-line fuel cell ohmic resistance measurement", Journal of Power Sources, vol. 160, (2006), pp. 1088-1095.

[11] K. S. Champlin and K. Bertness, "A fundamentally new approach to battery performance analysis using DFRATM/DFISTM technology", Twenty-second international telecommunications energy conference, (2000) Sep 10-14, Phoenix, United States.

[12] K. S. Champlin and K. Bertness, "Discrete frequency immittance spectroscopy (DFISTM) a new technique for battery instrumentation", Sixteenth annual battery conference on applications and advances, (2001) Jan 09-12, Calif State Univ Long Beach, Canada.

[13] S. R. Nelatury and P. Singh, "Equivalent circuit parameters of nickel metal hydride batteries from sparse impedance measurements", Journal of Power Sources, vol. 132, no. 1-2, (2004), pp. 309-314.

[14] P. Singh, S. Kaneria, J. Broadhead, X. Wang and J. Burdick, "Fuzzy logic estimation of SOH of $125 \mathrm{Ah}$ VRLA batteries", 26th Annual International Telecommunications Energy Conference, (2004) September 1923, Chicago,United states.

[15] J, Liguo, "Internal resistance detector of chargeable battery", Master thesis, University of Electronic Science and Technology of China, (2006).

[16] J. Fan and P. S. Fedkiw, "Electrochemical impedance spectra of full cells: Relation to capacity and capacity-rate of rechargeable $\mathrm{Li}$ cells using $\mathrm{LiCoO} 2$, LiMn2O4, and $\mathrm{LiNiO} 2$ cathodes", Journal of Power Sources, vol. 72, (1998), p p.165-173.

\section{Authors}

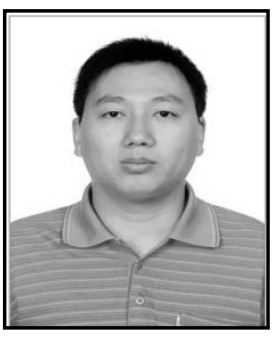

Zhang Hua, he received B.E. \& M.E. degrees in Measuring and Testing Technology and Instruments from the Northeast Petroleum University, China, in 2002 and 2009 respectively. Currently, he is a Ph.D. Candidate in Instrument Science and technology from the Harbin Institute of Technology, Harbin, China. His research interest covers energy management systems, and fault diagnosis of the battery. E-mail: zhxueju@163.com. 